\title{
Adaptive Wavelet BEM for Boundary Integral Equations: Theory and Numerical Experiments*
}

\author{
S. Dahlke, H. Harbrecht, M. Utzinger, Meimar ${ }^{\dagger}$
}

May 5, 2017

\begin{abstract}
We are concerned with the numerical treatment of boundary integral equations by means of the adaptive wavelet boundary element method (BEM). In particular, we consider the second kind Fredholm integral equation for the double layer potential operator on patchwise smooth manifolds contained in $\mathbb{R}^{3}$. The corresponding operator equations are treated by means of adaptive implementations that are in complete accordance with the underlying theory. The numerical experiments demonstrate that adaptive methods really pay off in this setting. The observed convergence rates fit together very well with the theoretical predictions based on the Besov regularity of the exact solution.
\end{abstract}

Keywords: Besov spaces, weighted Sobolev spaces, adaptive wavelet BEM, non-linear approximation, integral equations, double layer potential operator, regularity, manifolds.

AMS Subject Classification: 30H25, 35B65, 42C40, 45E99, 46E35, 47B38, $65 \mathrm{~T} 60$.

\footnotetext{
${ }^{*}$ This work has been supported by the Deutsche Forschungsgemeinschaft DFG (DA 360/19-1) and by the Swiss National Science Foundation SNSF (200021E-142224/1).

${ }^{\dagger}$ Corresponding author.
} 


\section{Introduction}

This paper is concerned with the theoretical analysis and numerical treatment of integral equations of the form

$$
S(u)=g \quad \text { on } \quad \partial \Omega
$$

where $\partial \Omega$ denotes a patchwise smooth boundary of some bounded domain $\Omega \subset \mathbb{R}^{d}$. A typical example is given by the second kind Fredholm integral equation

$$
S_{\mathrm{DL}}(u):=\left(\frac{1}{2} \mathrm{Id}-K\right)(u)=g \quad \text { on } \quad \partial \Omega
$$

where

$$
v \mapsto K(v):=\frac{1}{4 \pi} \int_{\partial \Omega} v(y) \frac{\partial}{\partial \eta(y)} \frac{1}{|\cdot-y|_{2}} \mathrm{~d} \sigma(y)
$$

denotes the harmonic double layer potential operator on $\partial \Omega$ which naturally arises from the so-called indirect method for Dirichlet problems for Laplace's equation in $\Omega$. For details and further references, see, e.g., [37, Chapter 3.4], as well as [7, 31, 32, 34, 41].

Indirect methods obviously provide a reduction of the problem dimension. Therefore, in recent years, much effort has been spent to design efficient numerical schemes for the solution to these kind of equations such as, e.g., the multipole method [35], the panel clustering [27] or the adaptive cross approximation [1]. In particular, one of the major computational bottlenecks is given by the fact that the discretization of (1) usually leads to densely populated matrices. In this regard, wavelet-based approaches provide striking advantages, since the wavelet's vanishing moment property can be used to design very powerful compression strategies $[13,38]$. Although these algorithms turned out to be quite successful when dealing with classical test problems, for real-world problems involving millions of unknowns, it is necessary to further enhance efficiency by means of adaptive strategies. In the meantime, fully adaptive wavelet methods which are guaranteed to converge with optimal order 
have been established $[12,25]$. Although reliable error estimators for boundary integral operators exist and optimal convergence of traditional boundary element discretizations have been proven, see, e.g., $[19,20,23]$, we are not aware of any other method which is also computationally optimal.

Although the numerical experiments performed so far have been quite promising, one principle problem remains. Besides numerical evidence, it is of course very desirable to derive rigorous statements under which conditions the use of adaptive algorithms for (1) really pays off in practice. Given a dictionary $\mathfrak{B}$ in the corresponding solution space, the best one could expect is that the adaptive algorithm realizes the convergence rate of the associated best $n$-term approximation with respect to $\mathfrak{B}$ which is defined as follows.

Definition 1.1. Let $\mathcal{G}$ denote a (quasi-) normed space and let $\mathfrak{B}=\left\{b_{1}, b_{2}, \ldots\right\}$ be some countable subset of $\mathcal{G}$. Then

$$
\sigma_{n}(u ; \mathfrak{B}, \mathcal{G}):=\inf _{i_{1}, \ldots, i_{n} \in \mathbb{N}} \inf _{c_{1}, \ldots, c_{n} \in \mathbb{C}}\left\|u-\sum_{m=1}^{n} c_{m} b_{i_{m}} \mid \mathcal{G}\right\|, \quad n \in \mathbb{N}
$$

defines the error of the best $n$-term approximation to some element $u$ with respect to the dictionary $\mathfrak{B}$ in the (quasi-) norm of $\mathcal{G}$.

Since $i_{1}, \ldots, i_{n}$ and $c_{1}, \ldots, c_{n}$ may depend on $u$ in an arbitrary way, this reflects how well we can approximate $u$ using a finite linear combination of elements in $\mathfrak{B}$. These linear combinations obviously form a highly non-linear manifold in the linear space $\mathcal{G}$. Of course, to get a reasonable result concerning the achievable rate of best $n$-term approximations, additional information (such as further smoothness properties) of the target function $u$ is needed. This is usually modeled by its membership in some additional (quasi-) normed space $\mathcal{F}$. Hence, if $\mathcal{F}$ denotes such a space which is 
embedded into $\mathcal{G}$, then we may study the asymptotic decay of

$$
\sigma_{n}(\mathcal{F} ; \mathfrak{B}, \mathcal{G}):=\sup _{\substack{u \in \mathcal{F},\|u \mid \mathcal{F}\| \leq 1}} \sigma_{n}(u ; \mathfrak{B}, \mathcal{G}), \quad \text { as } \quad n \rightarrow \infty
$$

If the dictionary consists of a wavelet basis, it is indeed possible to construct optimal adaptive algorithms. That is, these schemes are guaranteed to converge with optimal order (i.e., they realize the convergence rate of best $n$-term wavelet approximation as defined above), while their computational costs stay proportional to the used number of degrees of freedom $[4,5]$. Therefore, we can state that, in the wavelet setting, adaptivity really pays if the convergence order of best $n$-term approximation is strictly higher than the corresponding rate for classical non-adaptive algorithms. In this regard, it has been shown that the convergence order of (4) w.r.t. the $L_{2}$-norm is determined by the maximal regularity $\alpha$ of the $d$-variate function under consideration in the so-called adaptivity scale of Besov spaces

$$
B_{\tau}^{\alpha_{\tau}}\left(L_{\tau}\right) \quad \text { with } \quad \frac{1}{\tau}=\frac{\alpha_{\tau}}{d}+\frac{1}{2}, \quad 0<\alpha_{\tau}<\alpha
$$

where for all $\alpha_{\tau}>0$,

$$
\sigma_{n}\left(B_{\tau}^{\alpha_{\tau}}\left(L_{\tau}\right) ; \mathfrak{B}, L_{2}\right) \sim n^{-\alpha_{\tau} / d}, \quad \text { as } n \rightarrow \infty
$$

see, e.g., $[10,15,16]$. On the other hand, the convergence order of classical (uniform) algorithms is given by $n^{-s / d}$, where $s$ denotes the maximal regularity of the exact solution in the $L_{2}$-Sobolev scale, i.e., $s=\sup \left\{\mu>0 \mid u \in H^{\mu}\right\}$; see, e.g., $[8,10,26]$ for details. In conclusion, the use of adaptivity is justified if the smoothness $\alpha$ of the exact solution $u$ to (1) in the adaptivity scale (5) of Besov spaces is higher than its Sobolev regularity $s$.

For partial differential equations on bounded domains, a lot of positive results in this direction exist; see, e.g., [9]. Quite recently, in [11], also a positive result for integral operators on two- 
dimensional patchwise smooth manifolds has been derived. It has turned out that for a large class of operators, including the second kind Fredholm integral equation (2) for the double layer potential operator (3), the Besov smoothness of the solution in the adaptivity scale can be up to twice as high as the Sobolev regularity, so that adaptivity definitely makes sense.

Nevertheless, one important issue still has to be discussed. Of course, the estimate in (6) is of asymptotic nature, so that it is not clear whether the advantage of adaptivity can really be observed in numerical practice. For example, it might happen that some of the involved constants are so large that the asymptotic behavior becomes significant only for values of $n$ which are far beyond any practical feasibility. It is one of the major goals of this paper to convince the reader that this is actually not the case. In order to do so, we performed numerical experiments that go far beyond trivial toy problems. We considered the double layer potential operator on patchwise smooth manifolds. Our test cases, e.g., the Fichera vertex, are chosen in such a way that nontrivial singularities in the solution may show up at the interfaces. It turns out that the adaptive wavelet BEM indeed completely resolves all the singularities without any a priori information on the refinement strategy. We designed test cases where the solutions provably possess a relatively small Sobolev smoothness, but twice as much Besov regularity. And indeed, the adaptive wavelet algorithm converges twice as fast as the corresponding uniform scheme that simply uses all wavelets up to a given refinement level. Finally, we like to emphasize that our numerical realizations are in complete accordance with the theory development in $[12,25]$.

The paper is organized as follows: In Section 2, we start with some preparations concerning the parametrization of surfaces. In addition, here we define a scale of weighted Sobolev spaces $X_{\varrho}^{k}(\partial \Omega)$ which is needed to formulate our theoretical results. We also present a short introduction to the theory of layer potentials as far as it is needed for our purposes (see Subsection 2.2). Moreover, we discuss the basic properties of the wavelet bases that are required for the use in adaptive algorithms. In Section 3, we define Besov-type spaces $B_{\Psi, q}^{\alpha}\left(L_{p}(\partial \Omega)\right)$ as introduced in [11] and clarify their 
relations to best $n$-term wavelet approximation. Our main Besov regularity results for solutions to integral equations are briefly summarized in Subsection 3.2. Section 4 is dedicated to the adaptive wavelet method. We survey on the basic ingredients needed to realize an algorithm that realizes asymptotically optimal complexity. By asymptotically optimal we mean that any target accuracy can be achieved at a computational expense that stays proportional to the number of degrees of freedom that is needed to approximate the solution by $n$-term approximation with the same accuracy. Numerical results are then presented in Section 5. They are in good agreement with the theory. Finally, in Section 6, we state some concluding remarks.

Notation: For families $\left\{a_{i}\right\}_{i \in \mathcal{I}}$ and $\left\{b_{i}\right\}_{i \in \mathcal{I}}$ of non-negative real numbers over a common index set we write $a_{i} \lesssim b_{i}$ if there exists a constant $c>0$ such that

$$
a_{i} \leq c \cdot b_{i}
$$

holds uniformly in $i \in \mathcal{I}$. Consequently, $a_{i} \sim b_{i}$ means $a_{i} \lesssim b_{i}$ and $b_{i} \lesssim a_{i}$.

\section{Preliminaries}

\subsection{Surfaces $\partial \Omega$ and weighted Sobolev spaces $X_{\varrho}^{k}(\partial \Omega)$}

In this paper, we consider Lipschitz surfaces $\partial \Omega$ which are boundaries of bounded, simply connected, closed domains $\Omega \subset \mathbb{R}^{3}$ with polyhedral structure and finitely many quadrilateral sides. W.l.o.g. we can assume all these sides to be flat with corresponding straight edges; cf. [11, Remark 2.1].

We will pursue essentially two different (but equivalent) approaches to describe $\partial \Omega$, where both of them will be used later on. For the first approach, consider the patchwise decomposition

$$
\partial \Omega=\bigcup_{i=1}^{I} \overline{F_{i}}
$$


where $\overline{F_{i}}$ denotes the closure of the $i$ th (open) patch of $\partial \Omega$ which is a subset of some affine hyperplane in $\mathbb{R}^{3}$, bounded by a closed polygonal chain connecting exactly four points (vertices $\nu$ of $\Omega$ ). Here, we only require that the partition (7) is essentially disjoint in the sense that the intersection of any two patches $\overline{F_{i}} \cap \overline{F_{\ell}}, i \neq \ell$, is either empty, a common edge, or a common vertex of $\Omega$. Furthermore, we will assume the existence of (sufficiently smooth) diffeomorphic parametrizations

$$
\kappa_{i}:[0,1]^{2} \rightarrow \overline{F_{i}}, \quad i=1, \ldots, I
$$

which map the unit square onto these patches. Finally, we define the class of patchwise smooth functions on $\partial \Omega$ by

$$
C_{\mathrm{pw}}^{\infty}(\partial \Omega):=\left\{u: \partial \Omega \rightarrow \mathbb{C} \mid u \text { is globally continuous and }\left.u\right|_{\overline{F_{i}}} \in C^{\infty}\left(\overline{F_{i}}\right) \text { for all } i\right\} .
$$

In the second approach, the surface of $\Omega$ is modeled (locally) in terms of the boundary of its tangent cones $\mathcal{C}_{n}$, subordinate to the vertices $\nu_{1}, \ldots, \nu_{N}$ of $\Omega$. For $n \in\{1, \ldots, N\}$, the boundary of the infinite cone $\mathcal{C}_{n}$ consists of $T_{n} \geq 3$ essentially disjoint, open plane sectors (called faces) which will be denoted by $\Gamma^{n, 1}, \ldots, \Gamma^{n, T_{n}}$, i.e.,

$$
\partial \mathcal{C}_{n}=\bigcup_{t=1}^{T_{n}} \overline{\Gamma^{n, t}}, \quad n=1, \ldots, N
$$

It will be convenient to use local polar coordinates $(r, \phi)$ in each of these faces $\Gamma^{n, t}$. Then, every function $f_{n}: \partial \mathcal{C}_{n} \rightarrow \mathbb{C}$ can be described by a finite collection of functions $\left(f_{n, 1}, \ldots, f_{n, T_{n}}\right)$ of the variable $y:=(r \cos (\phi), r \sin (\phi)) \in \mathbb{R}^{2}$. Moreover, if $\gamma_{n, t}$ denotes the opening angle of $\Gamma^{n, t}$, the quantities $r$ and

$$
q(\phi):=\min \left\{\phi, \gamma_{n, t}-\phi\right\} \in(0, \pi)
$$


serve as a distance measure of the point $y$ to the face boundary; see [11, Formula (7)] for details. Therefore, following [18], weighted Sobolev spaces on the boundary of the cone $\mathcal{C}_{n}$ can be defined as the closure of all continuous, facewise smooth, compactly supported functions on $\partial \mathcal{C}_{n}$,

$$
C_{0, \mathrm{fw}}^{\infty}\left(\partial \mathcal{C}_{n}\right):=\left\{f_{n} \in C_{0}\left(\partial \mathcal{C}_{n}\right) \mid f_{n, t} \in C^{\infty}\left(\overline{\Gamma^{n, t}}\right) \text { for all } t=1, \ldots, T_{n}\right\}
$$

with respect to the norm $\left\|f_{n} \mid X_{\varrho}^{k}\left(\partial \mathcal{C}_{n}\right)\right\|$ given by

$$
\left\|f_{n}\left|L_{2}\left(\partial \mathcal{C}_{n}\right)\left\|+\sum_{t=1}^{T_{n}} \sum_{\substack{\beta=\left(\beta_{r}, \beta_{\phi}\right) \in \mathbb{N}_{0}^{2} \\ 1 \leq|\beta| \leq k}}\right\|\left(1+\frac{1}{r}\right)^{\varrho}(q r)^{\beta_{r}}\left(\frac{\partial}{\partial r}\right)^{\beta_{r}} q^{\beta_{\phi}-\varrho}\left(\frac{\partial}{\partial \phi}\right)^{\beta_{\phi}} f_{n, t}\right| L_{2}\left(\Gamma^{n, t}\right)\right\|
$$

That is, we let

$$
X_{\varrho}^{k}\left(\partial \mathcal{C}_{n}\right):=\overline{C_{0, \mathrm{fw}}^{\infty}\left(\partial \mathcal{C}_{n}\right)}\left\|\cdot \mid X_{\varrho}^{k}\left(\partial \mathcal{C}_{n}\right)\right\|
$$

where, as usual, the sum over an empty set is to be interpreted as zero, $k \in \mathbb{N}$ is the smoothness of the space, and $\varrho \in[0, k]$ controls the strength of the weight.

In order to analyze functions $u$ defined on the whole surface $\partial \Omega \subset \bigcup_{n=1}^{N} \partial \mathcal{C}_{n}$, we localize them with the help of a special resolution of unity $\left(\varphi_{n}\right)_{n=1}^{N}$ to cone faces near the vertices $\nu_{1}, \ldots, \nu_{N}$ of $\Omega$. Hence, for $u: \partial \Omega \rightarrow \mathbb{C}, k \in \mathbb{N}$, and $0 \leq \varrho \leq k$, we let

$$
\left\|u\left|X_{\varrho}^{k}(\partial \Omega)\left\|:=\sum_{n=1}^{N}\right\| \varphi_{n} u\right| X_{\varrho}^{k}\left(\partial \mathcal{C}_{n}\right)\right\|
$$

and define the weighted Sobolev space on $\partial \Omega$ as

$$
X_{\varrho}^{k}(\partial \Omega):=\overline{C_{\mathrm{pw}}^{\infty}(\partial \Omega)}\left\|\cdot \mid X_{\varrho}^{k}(\partial \Omega)\right\| .
$$

For details, the interested reader is again referred to [11]. 


\section{$2.2 \quad$ Layer potentials}

We shall be concerned with the solution of the Dirichlet problem for the Laplacian

$$
\Delta U=0 \quad \text { in } \Omega, \quad U=g \text { on } \partial \Omega
$$

on a domain $\Omega \subset \mathbb{R}^{3}$ with patchwise smooth boundary $\partial \Omega$ by means of harmonic double layer potentials. To that end, let $\sigma$ denote the canonical surface measure. Since this surface is assumed to be Lipschitz, for $\sigma$-a.e. $x \in \partial \Omega$ there exists the outward pointing normal vector $\eta(x)$. By $\partial / \partial \eta(x)$ we denote the corresponding conormal derivative in $x \in \partial \Omega$. Making the potential ansatz

$$
U(x):=\frac{1}{4 \pi} \int_{\partial \Omega} u(y) \frac{\partial}{\partial \eta(y)} \frac{1}{|x-y|_{2}} \mathrm{~d} \sigma(y), \quad x \in \Omega
$$

and letting $x$ tend to $\partial \Omega$, we arrive, in view of the jump condition, at the second kind Fredholm integral equation (2) for the unknown density $u$. The integral operator under consideration is of order zero,

$$
S_{\mathrm{DL}}=\left(\frac{1}{2} \mathrm{Id}-K\right): L_{2}(\partial \Omega) \rightarrow L_{2}(\partial \Omega)
$$

where Id denotes the identical mapping on $\partial \Omega$ and $K$ is the harmonic double layer potential operator as defined in (3). One speaks here of the indirect method: The sought solution $U \in H^{1}(\Omega)$ is not computed directly, but indirectly via the evaluation of the potential (10), as soon as the potential's density $u$ is known.

When it comes to the numerical approximation of the solution $u$ to (2), well-posedness of the problem is essential. Invertibility within the (unweighted) Sobolev scale $H^{s}(\partial \Omega)$ is known as Verchota's Theorem [41]; see also [18, Remark A.5].

Proposition 2.1 (Verchota [41, Theorem 3.3(iii)]). For all $s \in[0,1]$, the bounded linear operator 
$S_{\mathrm{DL}}: H^{s}(\partial \Omega) \rightarrow H^{s}(\partial \Omega)$ is invertible.

However, for our purposes, bounded invertibility in $H^{s}(\partial \Omega)$ is not enough. As we shall see later in Subsection 3.2, also regularity estimates in the weighted Sobolev scale $X_{\varrho}^{k}(\partial \Omega)$ as introduced in Subsection 2.1 are needed. In this context, the subsequent result taken from [18] is particularly useful.

Proposition 2.2 (Elschner [18, Remark 4.3]). There exists a constant $\varrho_{0} \in(1,3 / 2)$, depending on the surface $\partial \Omega$, such that the following is true: For all $0 \leq \varrho<\varrho_{0}$ and every $k \in \mathbb{N}$ with $\varrho \leq k$, the bounded linear operator $S_{\mathrm{DL}}: X_{\varrho}^{k}(\partial \Omega) \rightarrow X_{\varrho}^{k}(\partial \Omega)$ is invertible.

Note that in the notation of [41] $K$ is replaced by $-K$ and that the operator $K$ considered in [18] differs from our notation by a factor of $1 / 2$. Nevertheless, the whole analysis carries over.

\subsection{Wavelet bases}

During the past years, wavelets on domains $\Omega \subseteq \mathbb{R}^{d}$ have become a powerful tool in both, pure and applied mathematics. More recently, several authors proposed various constructions of wavelet systems extending the idea of multiscale analysis to manifolds based on patchwise descriptions such as (7); see, e.g., [2, 3, 6, 14, 28, 29]. Later on in this paper, bases of these kinds will be used to define new types of Besov spaces on $\partial \Omega$. Therefore, in this subsection, we collect some basic properties that will be needed for this purpose.

With the help of the parametric liftings $\kappa_{i}, i=1, \ldots, I$, an inner product for functions $u, v: \partial \Omega \rightarrow$ $\mathbb{C}$ can be defined patchwise by

$$
\langle u, v\rangle:=\sum_{i=1}^{I}\left\langle u \circ \kappa_{i}, v \circ \kappa_{i}\right\rangle_{\square},
$$

where $\langle\cdot, \cdot\rangle_{\square}$ denotes the usual $L_{2}$-inner product on the square $[0,1]^{2}$. Since all $\kappa_{i}$ are assumed to be 
sufficiently smooth the norm induced by $\langle\cdot, \cdot\rangle$ can be shown to be equivalent to the norm in $L_{2}(\partial \Omega)$ :

$$
\|\cdot\|\left\|_{0}:=\sqrt{\langle\cdot, \cdot\rangle} \sim\right\| \cdot \mid L_{2}(\partial \Omega) \|,
$$

see, e.g., formula (4.5.3) in [14].

Most of the known wavelet constructions are based on tensor products of boundary-adapted wavelets/scaling functions (defined on intervals) which are finally lifted to the patches $F_{i}$ describing the surface $\partial \Omega$. A wavelet basis $\Psi=\left(\Psi^{\partial \Omega}, \widetilde{\Psi}^{\partial \Omega}\right)$ on $\partial \Omega$ then consists of two collections of functions $\psi_{j, \xi}^{\partial \Omega}$ and $\widetilde{\psi}_{j, \xi}^{\partial \Omega}$, respectively, that form $(\langle\cdot, \cdot\rangle$-biorthogonal $)$ Riesz bases for $L_{2}(\partial \Omega)$. In particular, every $u \in L_{2}(\partial \Omega)$ has a unique expansion

$$
u=P_{j^{\star}-1}(u)+\sum_{j \geq j^{\star}} \sum_{\xi \in \nabla_{j}^{\partial \Omega}}\left\langle u, \widetilde{\psi}_{j, \xi}^{\partial \Omega}\right\rangle \psi_{j, \xi}^{\partial \Omega}
$$

satisfying

$$
\left\|u\left|L_{2}(\partial \Omega)\|\sim\| P_{j^{\star}-1}(u)\right| L_{2}(\partial \Omega)\right\|+\left(\sum_{j \geq j^{\star}} \sum_{\xi \in \nabla_{j}^{\partial \Omega}}\left|\left\langle u, \widetilde{\psi}_{j, \xi}^{\partial \Omega}\right\rangle\right|^{2}\right)^{1 / 2} .
$$

Therein, $P_{j^{\star}-1}$ denotes the biorthogonal projector that maps $L_{2}(\partial \Omega)$ onto the finite dimensional span of all generators on the coarsest level $j^{\star}-1$.

In the sequel, we will require that the wavelet basis under consideration satisfies all the conditions collected in the following assumption.

\section{Assumption 2.3.}

(I) As indicated in (12), both (the primal and the dual) systems are indexed by their level of resolution $j \geq j^{\star}$, as well as their location (and type) $\xi \in \nabla^{\partial \Omega}$. We assume that this collection 
of grid points on the surface $\partial \Omega$ can be split up according to the levels $j$ and the patches $\overline{F_{i}}$ :

$$
\nabla^{\partial \Omega}=\bigcup_{j=j^{\star}}^{\infty} \nabla_{j}^{\partial \Omega}, \quad \text { where, for all } j \geq j^{\star}, \quad \nabla_{j}^{\partial \Omega}=\bigcup_{i=1}^{I} \nabla_{j}^{F_{i}} \quad \text { with } \quad \# \nabla_{j}^{F_{i}} \sim 2^{2 j}
$$

(II) All dual wavelets are $L_{2}$-normalized:

$$
\left\|\widetilde{\psi}_{j, \xi}^{\partial \Omega} \mid L_{2}(\partial \Omega)\right\| \sim 1 \quad \text { for all } \quad j \geq j^{\star}, \xi \in \nabla_{j}^{\partial \Omega}
$$

(III) We assume that all elements $\widetilde{\psi}_{j, \xi}^{\partial \Omega} \in \widetilde{\Psi}^{\partial \Omega}$ are compactly supported on $\partial \Omega$. Furthermore, we assume that their supports contain the corresponding grid point $\xi$ and satisfy

$$
\left|\operatorname{supp} \widetilde{\psi}_{j, \xi}^{\partial \Omega}\right| \sim 2^{-2 j} \quad \text { for all } \quad j \geq j^{\star}, \xi \in \nabla_{j}^{\partial \Omega}
$$

(IV) Consider the set $\Pi_{\widetilde{d}-1}\left([0,1]^{2}\right)$ of polynomials $\mathcal{P}$ on the unit square which have a total degree $\operatorname{deg} \mathcal{P}$ strictly less than $\widetilde{d}$. Then, we assume that the dual system $\widetilde{\Psi}^{\partial \Omega}$ satisfies

$$
\left\langle\mathcal{P}, \widetilde{\psi}_{j, \xi}^{\partial \Omega} \circ \kappa_{i}\right\rangle_{\square}=0 \quad \text { for all } \quad \mathcal{P} \in \Pi_{\widetilde{d}-1}\left([0,1]^{2}\right),
$$

whenever $\widetilde{\psi}_{j, \xi}^{\partial \Omega} \in \widetilde{\Psi}^{\partial \Omega}$ is completely supported in the interior of some patch $F_{i} \subset \partial \Omega, i \in$ $\{1, \ldots, I\}$. This property is commonly known as vanishing moment property of order $\widetilde{d} \in \mathbb{N}$.

(V) The number of dual wavelets at level $j$ with distance $2^{-j}$ to one of the patch boundaries is of order $2^{j}$, i.e.,

$$
\#\left\{\xi \in \nabla_{j}^{\partial \Omega} \mid 0<\operatorname{dist}\left(\operatorname{supp} \widetilde{\psi}_{j, \xi}^{\partial \Omega}, \bigcup_{i=1}^{I} \partial F_{i}\right) \lesssim 2^{-j}\right\} \sim 2^{j} \quad \text { for all } \quad j \geq j^{\star} .
$$


Moreover, for the dual wavelets intersecting one of the patch interfaces, we assume that

$$
\#\left\{\xi \in \nabla_{j}^{\partial \Omega} \mid \operatorname{supp} \widetilde{\psi}_{j, \xi}^{\partial \Omega} \cap \bigcup_{i=1}^{I} \partial F_{i} \neq \emptyset\right\} \lesssim 2^{j} \quad \text { for all } \quad j \geq j^{\star}
$$

(VI) Every point $x \in \partial \Omega$ is contained in the supports of a uniformly bounded number of dual wavelets at level $j$ :

$$
\#\left\{\xi \in \nabla_{j}^{\partial \Omega} \mid x \in \operatorname{supp} \widetilde{\psi}_{j, \xi}^{\partial \Omega}\right\} \lesssim 1 \quad \text { for all } \quad j \geq j^{\star} \quad \text { and each } \quad x \in \partial \Omega \text {. }
$$

(VII) Finally, we assume that the Sobolev spaces $H^{s}(\partial \Omega)=W^{s}\left(L_{2}(\partial \Omega)\right)$ in the scale

$$
-\frac{1}{2}<s<\min \left\{\frac{3}{2}, s_{\partial \Omega}\right\}
$$

can be characterized by the decay of wavelet expansion coefficients, that is

$$
\left\|u\left|H^{s}(\partial \Omega)\|\sim\| P_{j^{\star}-1}(u)\right| L_{2}(\partial \Omega)\right\|+\left(\sum_{j \geq j^{\star}} \sum_{\xi \in \nabla_{j}^{\partial \Omega}} 2^{2 s j}\left|\left\langle u, \widetilde{\psi}_{j, \xi}^{\partial \Omega}\right\rangle\right|^{2}\right)^{1 / 2} .
$$

Here, the spaces for negative $s$ are defined by duality and $s_{\partial \Omega} \geq 1$ depends on the interior angles between different patches $F_{i}$ of the manifold under consideration; cf. [14, Section 4.5].

Fortunately, all these assumptions are satisfied for all the constructions we mentioned at the beginning of this subsection. In particular, the composite wavelet basis as constructed in [14] is a typical example which will serve as our main reference. Note that although those wavelets are usually at most continuous across patch interfaces, they are able to capture arbitrary high smoothness in the interior by increasing the order of the underlying boundary-adapted wavelets. 


\section{Besov regularity}

\subsection{Besov-type function spaces on $\partial \Omega$}

Besov spaces essentially generalize the concept of Sobolev spaces. On $\mathbb{R}^{d}$ they are typically defined using harmonic analysis, finite differences, moduli of smoothness, or interpolation techniques. Characteristics (embeddings, interpolation results, and approximation properties) of these scales of spaces are then obtained by reducing the assertion of interest to the level of sequences spaces by means of characterizations in terms of building blocks (atoms, local means, quarks, or wavelets). To mention at least a few references, the reader is referred to the monographs [36, 39], as well as to the articles $[16,21,33]$. This list is clearly not complete.

Besov spaces on manifolds such as boundaries of domains in $\mathbb{R}^{d}$ can be defined as trace spaces or via pullbacks based on (overlapping) resolutions of unity. In general, traces of wavelets are not wavelets anymore, and if we use pullbacks, then wavelet characterizations are naturally limited by the global smoothness of the underlying manifold. Therefore, let us recall a notion of Besov-type spaces from [11, Definition 4.1] which is based on expansions w.r.t. some biorthogonal wavelet Riesz

basis $\Psi=\left(\Psi^{\partial \Omega}, \widetilde{\Psi}^{\partial \Omega}\right)$ satisfying the conditions of the previous section which we assume to be given fixed:

Definition 3.1. A tuple of real parameters $(\alpha, p, q)$ is said to be admissible if

$$
\frac{1}{2} \leq \frac{1}{p} \leq \frac{\alpha}{2}+\frac{1}{2} \quad \text { and } \quad 0<q \leq \begin{cases}2 & \text { if } 1 / p=\alpha / 2+1 / 2 \\ \infty, & \text { otherwise }\end{cases}
$$

Given a wavelet basis $\Psi=\left(\Psi^{\partial \Omega}, \widetilde{\Psi}^{\partial \Omega}\right)$ on $\partial \Omega$ and a tuple of admissible parameters $(\alpha, p, q)$ let $B_{\Psi, q}^{\alpha}\left(L_{p}(\partial \Omega)\right)$ denote the collection of all complex-valued functions $u \in L_{2}(\partial \Omega)$ such that the (quasi-) 
norm

$$
\left\|u\left|B_{\Psi, q}^{\alpha}\left(L_{p}(\partial \Omega)\right)\|:=\| P_{j^{\star}-1}(u)\right| L_{p}(\partial \Omega)\right\|+\left(\sum_{j \geq j^{\star}} 2^{j\left(\alpha+2\left[\frac{1}{2}-\frac{1}{p}\right]\right) q}\left[\sum_{\xi \in \nabla_{j}^{\partial \Omega}}\left|\left\langle u, \widetilde{\psi}_{j, \xi}^{\partial \Omega}\right\rangle\right|^{p}\right]^{q / p}\right)^{1 / q}
$$

is finite (with the usual modification if $q=\infty$ ).

In the remainder of this subsection, we collect some basic properties of the Besov-type spaces introduced above. To start with, we note that all spaces $B_{\Psi, q}^{\alpha}\left(L_{p}(\partial \Omega)\right)$ are quasi-Banach spaces, Banach spaces if and only if $\min \{p, q\} \geq 1$, and Hilbert spaces if and only if $p=q=2$.

Formally, different bases $\Psi$ might lead to different function spaces even if all remaining parameters $(\alpha, p, q)$ that determine the spaces may coincide. Nevertheless, in [42], it has been shown that under very natural conditions the resulting Besov spaces coincide up to equivalent norms. These conditions are fortunately satisfied by most of the wavelet bases which are available in the literature.

Finally, let us recall an assertion which clarifies the relation of the scales $B_{\Psi, q}^{\alpha}\left(L_{p}(\partial \Omega)\right)$ and best $n$-term wavelet approximation; cf. [11, Proposition 4.7]. For a visualization of the involved embeddings we refer to the left DeVore-Triebel diagram in Figure 1.

Proposition 3.2. For $\gamma \in \mathbb{R}$, let $\left(\alpha+\gamma, p_{0}, q_{0}\right)$ and $\left(\alpha, p_{1}, q_{1}\right)$ be admissible parameter tuples. If $\gamma>2 \cdot \max \left\{0, \frac{1}{p_{0}}-\frac{1}{p_{1}}\right\}$, then

$$
\sigma_{n}\left(B_{\Psi, q_{0}}^{\alpha+\gamma}\left(L_{p_{0}}(\partial \Omega)\right) ; \Psi^{\partial \Omega}, B_{\Psi, q_{1}}^{\alpha}\left(L_{p_{1}}(\partial \Omega)\right)\right) \sim n^{-\gamma / 2}
$$

Moreover, if $\gamma=2 \cdot \max \left\{0, \frac{1}{p_{0}}-\frac{1}{p_{1}}\right\}$ and $q_{0} \leq q_{1}$, then

$$
\sigma_{n}\left(B_{\Psi, q_{0}}^{\alpha+\gamma}\left(L_{p_{0}}(\partial \Omega)\right) ; \Psi^{\partial \Omega}, B_{\Psi, q_{1}}^{\alpha}\left(L_{p_{1}}(\partial \Omega)\right)\right) \sim n^{-\min \left\{\gamma / 2,1 / q_{0}-1 / q_{1}\right\}}
$$


In view of our application to integral equations, we are particularly interested in the rate of convergence of best $n$-term wavelet approximation to solutions $u \in B_{\Psi, \tau}^{s^{\prime}+\gamma}\left(L_{\tau}(\partial \Omega)\right)$ with

$$
s^{\prime} \in\left[0, \max \left\{3 / 2, s_{\partial \Omega}\right\}\right), \quad \gamma \geq 0, \quad \text { and } \quad \tau:=(\gamma / 2+1 / 2)^{-1}
$$

w.r.t. the norm in $H^{s^{\prime}}(\partial \Omega)$.

Corollary 3.3. For $s^{\prime}, \gamma$, and $\tau$ given by (20), we have

$$
\sigma_{n}\left(B_{\Psi, \tau}^{s^{\prime}+\gamma}\left(L_{\tau}(\partial \Omega)\right) ; \Psi^{\partial \Omega}, H^{s^{\prime}}(\partial \Omega)\right) \sim n^{-\gamma / 2} \quad \text { as } \quad n \rightarrow \infty
$$

Proof. Apply Proposition 3.2 with $p_{0}:=q_{0}:=\tau, p_{1}:=q_{1}:=2$, and $\alpha:=s^{\prime}$.

\subsection{Main regularity results}

Throughout the whole section, $\partial \Omega$ denotes the patchwise smooth boundary of some three-dimensional domain $\Omega$, as described in Subsection 2.1. Moreover, we assume to be given a biorthogonal wavelet Riesz basis $\Psi=\left(\Psi^{\partial \Omega}, \widetilde{\Psi}^{\partial \Omega}\right)$ on $\partial \Omega$, satisfying the requirements stated in Assumption 2.3.

Given an operator $S$ and a right-hand side $g: \partial \Omega \rightarrow \mathbb{C}$, we like to solve the equation

$$
S(u)=g \quad \text { on } \quad \partial \Omega
$$

for $u: \partial \Omega \rightarrow \mathbb{C}$. In particular, we are interested in the asymptotic behavior of the error of best $n$-term wavelet approximation to $u$, measured in the norm of $H^{s^{\prime}}(\partial \Omega)$ for some $s^{\prime} \geq 0$.

Theorem 3.4 ([11, Theorem 5.6]). Assume $\widetilde{d} \in \mathbb{N}, k \in\{1,2, \ldots, \widetilde{d}\}$, as well as $\varrho \in(0, k)$, and let $(s, p, p)$ be an admissible tuple of parameters with $s>0$. Whenever the solution $u$ to (21) is contained in the intersection of $B_{\Psi, p}^{s}\left(L_{p}(\partial \Omega)\right)$ and $X_{\varrho}^{k}(\partial \Omega)$, then it also belongs to the Besov-type 
space $B_{\Psi, \tau}^{\alpha}\left(L_{\tau}(\partial \Omega)\right)$ for all tuples $(\alpha, \tau, \tau)$ with

$$
\frac{1}{\tau}=\frac{\alpha}{2}+\frac{1}{2} \quad \text { and } \quad 0 \leq \alpha<2 \alpha^{\star}, \quad \text { where } \quad \alpha^{\star}=\min \left\{\varrho, k-\varrho, s-\left(\frac{1}{p}-\frac{1}{2}\right)\right\}
$$

Moreover, for every $0 \leq s^{\prime}<\min \left\{3 / 2, s_{\partial \Omega}\right\}$, satisfying

$$
s-s^{\prime} \geq 2\left(\frac{1}{p}-\frac{1}{2}\right)
$$

we have $\sigma_{n}\left(u ; \Psi^{\partial \Omega}, H^{s^{\prime}}(\partial \Omega)\right) \lesssim n^{-\gamma / 2}$ as $n \rightarrow \infty$ for all $\gamma<\gamma^{\star}$, where

$$
\gamma^{\star}:=s-s^{\prime}+\Theta \cdot\left(2 \alpha^{\star}-s\right) \geq 0 \quad \text { and } \quad \Theta:=1-\frac{s^{\prime}}{s-2(1 / p-1 / 2)} \in[0,1] \text {. }
$$

The second DeVore-Triebel diagram in Figure 1 illustrates a special case of Theorem 3.4. There, we have chosen $p=2$ and $0<s^{\prime}<s<\min \left\{\varrho, k-\varrho, 3 / 2, s_{\partial \Omega}\right\}$, such that particularly $\alpha^{\star}=s$ and $B_{\Psi, p}^{s}\left(L_{p}(\partial \Omega)\right)=H^{s}(\partial \Omega)$. The dotted line corresponds to the scale of spaces $B_{\Psi, p_{\Theta}}^{\alpha_{\Theta}}\left(L_{p_{\Theta}}(\partial \Omega)\right)$ which can be reached by complex interpolation of $H^{s}(\partial \Omega)$ and $B_{\Psi, \tau}^{\alpha}\left(L_{\tau}(\partial \Omega)\right)$. Interpolation is necessary since only those spaces which belong to the shaded area can be embedded into $H^{s^{\prime}}(\partial \Omega)$. For this special choice of the parameters we obtain that $u \in H^{s}(\partial \Omega) \cap X_{\varrho}^{k}(\partial \Omega)$ can be approximated in the norm of $H^{s^{\prime}}(\partial \Omega)$ at a rate arbitrarily close to $\gamma^{\star} / 2=s-s^{\prime}$, whereas the rate of convergence for best $n$-term wavelet approximation to an arbitrary function $u \in H^{s}(\partial \Omega)$ is $\left(s-s^{\prime}\right) / 2$; see Proposition 3.2. Hence, incorporating the additional knowledge about weighted Sobolev regularity (membership in $\left.X_{\varrho}^{k}(\partial \Omega)\right)$ allows to improve the rate of convergence up to a factor of two.

For the special case of the double layer operator $S:=S_{\mathrm{DL}}$, we obtain the following theorem. Its proof is based on a combination of Propositions 2.1 and 2.2 with Theorem 3.4; see [11].

Theorem 3.5 ([11, Theorem 5.8]). Let $s \in(0,1)$, as well as $k \in \mathbb{N}$, and $\varrho \in\left(0, \min \left\{\varrho_{0}, k\right\}\right)$ for 
some $\varrho_{0} \in(1,3 / 2)$ depending on the surface $\partial \Omega$. Moreover let $\alpha$ and $\tau$ be given such that

$$
\frac{1}{\tau}=\frac{\alpha}{2}+\frac{1}{2} \quad \text { and } \quad 0 \leq \alpha<2 \cdot \min \{\varrho, k-\varrho, s\}
$$

and let the Besov-type space $B_{\Psi, \tau}^{\alpha}\left(L_{\tau}(\partial \Omega)\right)$ be constructed with the help of a wavelet basis $\Psi=$ $\left(\Psi^{\partial \Omega}, \widetilde{\Psi}^{\partial \Omega}\right)$ possessing vanishing moments of order $\widetilde{d} \geq k$. Then, for every right-hand side $g \in H^{s}(\partial \Omega) \cap X_{\varrho}^{k}(\partial \Omega)$, the double layer equation (2) has a unique solution $u \in B_{\Psi, \tau}^{\alpha}\left(L_{\tau}(\partial \Omega)\right)$. Furthermore, if $s^{\prime} \in[0, s]$, then the error of the best $n$-term wavelet approximation to $u$ in the norm of $H^{s^{\prime}}(\partial \Omega)$ satisfies

$$
\sigma_{n}\left(u ; \Psi^{\partial \Omega}, H^{s^{\prime}}(\partial \Omega)\right) \lesssim n^{-\gamma / 2} \quad \text { for all } \quad \gamma<2 \cdot\left(1-\frac{s^{\prime}}{s}\right) \cdot \min \{\varrho, k-\varrho, s\}
$$

\section{Adaptive wavelet methods for integral equations}

Adaptive wavelet methods rely on an iterative solution method for the continuous boundary integral equation (1) under consideration, expanded with respect to the wavelet basis. To this end, we renormalize the wavelet basis $\Psi$ w.r.t. the underlying energy space. Then (1) is equivalent to the well-posed problem of finding $u=\Psi^{\partial \Omega} \mathbf{u}$ such that the infinite dimensional system of linear equations

$$
\mathbf{S u}=\mathbf{f}, \quad \text { where } \quad \mathbf{S}:=\left\langle S\left(\Psi^{\partial \Omega}\right), \Psi^{\partial \Omega}\right\rangle \quad \text { and } \quad \mathbf{f}:=\left\langle f, \Psi^{\partial \Omega}\right\rangle
$$

holds. For approximately solving this infinite dimensional system of linear equations, one has to perform matrix-vector multiplications by means of adaptive applications of the matrix $\mathbf{S}$ under consideration. The basic building blocks CoArse, Apply, Rhs, and Solve, which are needed to arrive at an adaptive algorithm of optimal complexity, have been introduced in $[4,5]$. Our particular implementation is based on piecewise constant wavelets as outlined in [13, 30], see also [25] for 
related results. In particular, we restrict the set of active wavelet functions to tree constraints which ensures the method's efficient implementation. Notice that the piecewise constant wavelets we use here are discontinuous. This implies that the norm equivalence (18) only holds in the range $-1 / 2<s<1 / 2$. Nevertheless, this limitation in the basis functions' smoothness does not change the rates of the best $n$-term approximations in the range of Sobolev spaces which can be characterized.

The specific adaptive algorithm we use has been proposed in $[22,24]$ and is similar to classical methods which consist of the following steps:$$
\text { Solve } \rightarrow \text { Estimate } \rightarrow \text { MARK } \rightarrow \text { Refine }
$$

For a given (finite) index set $\mathcal{T} \subset \nabla^{\partial \Omega}$, we solve the Galerkin system $(23)$ via $\mathbf{u}_{\mathcal{T}}=\operatorname{SoLVE}[\mathcal{T}]$. Then we estimate the (infinite) residuum $\mathbf{r}:=\mathbf{f}-\mathbf{S u}_{\mathcal{T}}$ with sufficient accuracy $\delta>0$ by computing

$$
\mathbf{r}_{\mathcal{T}^{\prime}}=\operatorname{RHS}[\delta / 2]-\operatorname{APPLY}\left[\delta / 2, \mathbf{u}_{\mathcal{T}}\right]
$$

relative to a finite index set $\mathcal{T} \subset \mathcal{T}^{\prime} \subset \nabla^{\partial \Omega}$ such that

$$
\left\|\mathbf{r}-\mathbf{r}_{\mathcal{T}^{\prime}}\right\|_{2} \leq \delta
$$

Herein, $\operatorname{RHS}[\delta / 2]$ produces a finitely supported approximation of the right-hand side with accuracy $\delta / 2$ and $\operatorname{APPLy}\left[\delta / 2, \mathbf{u}_{\mathcal{T}}\right]$ approximates the matrix-vector product $\mathbf{S u}_{\mathcal{T}}$ with accuracy $\delta / 2$. In order to have $\left\|\mathbf{r}_{\mathcal{T}^{\prime}}\right\|_{2}$ proportional to $\|\mathbf{r}\|_{2}$, i.e.,

$$
(1-\omega)\left\|\mathbf{r}_{\mathcal{T}^{\prime}}\right\|_{2} \leq\|\mathbf{r}\|_{2} \leq(1+\omega)\left\|\mathbf{r}_{\mathcal{T}^{\prime}}\right\|_{2}
$$


for fixed $0<\omega<1$, we apply the following iteration for some initial precision $\delta_{\text {init }}$ :

$$
\begin{aligned}
& \text { set } \delta=\delta_{\text {init }} \\
& \text { do } \\
& \quad \text { set } \delta=\delta / 2 \\
& \quad \text { calculate } \mathbf{r}_{\mathcal{T}^{\prime}}=\operatorname{RHS}[\delta / 2]-\operatorname{APPLY}\left[\delta / 2, \mathbf{u}_{\mathcal{T}}\right] \\
& \text { until } \delta \leq \omega\left\|\mathbf{r}_{\mathcal{T}^{\prime}}\right\|_{2}
\end{aligned}
$$

The until-clause $\delta \leq \omega\left\|\mathbf{r}_{\mathcal{T}^{\prime}}\right\|_{2}$ causes that this iteration terminates when (24) holds.

The supporting index set $\mathcal{T}^{\prime}$ of the approximate residuum $\mathbf{r}_{\mathcal{T}^{\prime}}$ enlarges the original index set $\mathcal{T}$ enough to ensure that the Galerkin solution with respect to $\mathcal{T}^{\prime}$ would reduce the error by a constant factor. Nevertheless, to control the complexity, we have to coarsen the index set $\mathcal{T}^{\prime}$ such that

$$
\left\|\mathbf{r}_{\mathcal{T}^{\prime \prime}}\right\|_{2} \leq \theta\left\|\mathbf{r}_{\mathcal{T}^{\prime}}\right\|_{2}
$$

for fixed $0<\theta<1$ sufficiently small. This is done by calling

$$
\mathbf{r}_{\mathcal{T}^{\prime \prime}}=\operatorname{COARSE}\left[\theta, \mathbf{r}_{\mathcal{T}^{\prime}}\right]
$$

It combines the steps mark and refine since the new index set $\mathcal{T}^{\prime \prime}$ enlarges the original index set $\mathcal{T}$ which corresponds to mesh refinement. We emphasize that $\mathcal{T}^{\prime \prime}$ is still large enough to guarantee the convergence of the algorithm when starting the procedure again with $\mathcal{T}:=\mathcal{T}^{\prime \prime}$. For all the details of the particular implementation, we refer the reader to [40]. 


\section{$5 \quad$ Numerical results}

\subsection{Right-hand side with point singularity}

We will present results for the Laplace equation solved by the second kind Fredholm integral equation (2) for the double layer potential operator (3). We choose Fichera's vertex as domain under consideration, i.e., $\Omega:=(0,1)^{3} \backslash(0,0.5]^{3}$. Its surface is parametrized by 12 patches in accordance with Subsection 2.1. We consider non-smooth Dirichlet data of the form

$$
g(x):=|x-(0.5,0.5,0.5)|^{-\alpha}
$$

for $\alpha=0.5$ and $\alpha=0.75$. These Dirichlet data admit a point singularity in the reentrant corner $(0.5,0.5,0.5)$ of the Fichera vertex.

According to the subsequent Theorem 5.1, for these right-hand sides we can expect a rate of convergence of least $n^{-(1-\alpha)}$ when using an optimal adaptive scheme. In contrast, we expect only half the rate, i.e., $n^{-(1-\alpha) / 2}$, when using uniform refinement.

Theorem 5.1. Let $\partial \Omega \subset \mathbb{R}^{3}$ denote a Lipschitz surface according to Subsection 2.1, assume $\nu$ to be one of its vertices, and let $1 / 2 \leq \alpha<1$. Moreover, denote by $u$ the unique solution to (2) with right-hand side given by

$$
g(x):=|x-\nu|^{-\alpha}, \quad x \in \partial \Omega .
$$

Then the error of the best $n$-term wavelet approximation to $u$ w.r.t. $L_{2}(\partial \Omega)$ converges at least at a rate of $n^{-(\alpha-1)}$, while the corresponding rate for uniform approximation is limited to $n^{-(1-\alpha) / 2}$.

Proof. From Proposition A.2 in the Appendix we have that $\sup \left\{s>0 \mid g \in H^{s}(\partial \Omega)\right\}=1-\alpha$ and thus Proposition 2.1 implies that the same upper bound holds for the solution $u$. In turn, this proves the limitation for the rate of convergence using uniform refinement. On the other hand, 
Proposition A.2 also yields that we can apply Theorem 3.5 with $k:=1$ and $s:=\varrho:=1-\alpha-\varepsilon$, where $\varepsilon>0$ can be chosen arbitrarily small, as well as $s^{\prime}:=0$. This shows that the best $n$-term rate is at least twice as high which completes the proof.

In Figure 2, the observed convergence rates are found. We plotted the energy norm of the residual vector for both, uniform and adaptive refinement, into a log-log plot. Notice that the results of the uniform refinement are produced by the same adaptive wavelet method via enforcing a uniform (hence non-adaptive) refinement in the step REFINE.

For $\alpha=0.5$ and uniform refinement, the left plot in Figure 2 shows a rate that seems to be even slightly worse than $n^{-(1-\alpha) / 2}=n^{-0.25}$. In case of adaptive refinement, we obtain a rate of $n^{-(1-\alpha)}=n^{-0.5}$, which is exactly what we expect. Another observation can be made by comparing the number of degrees of freedom which are necessary in order to compute the approximate density for uniform refinement and for adaptive refinement. The norm of the residual is about $6 \cdot 10^{-2}$ for uniform refinement with more than 3 million degrees of freedom. For adaptive refinement, we obtain a norm of the residual of about $4.2 \cdot 10^{-2}$ already for approximately 40000 degrees of freedom, which is quite impressive.

We find the approximate density in the left image of Figure 3, while the refinement produced by the adaptive wavelet scheme is shown right next to it. The refinement is visualized by plotting the indices of all active wavelets. It is clearly visible that the adaptive method refines towards reentrant corner, where the right-hand side has its singularity. We also observe an interesting pattern in form of a grid in the refinement around the corner. This artefact comes from the large support of the wavelets.

Next, let us discuss the numerical results for $\alpha=0.75$. Due to Theorem 5.1, for this choice, we expect the adaptive wavelet method to converge at a rate of at least $n^{-(1-\alpha)}=n^{-0.25}$. In contrast, for uniform refinement, we again only expect at most half the rate, i.e., $n^{-(1-\alpha) / 2}=n^{-0.125}$. Indeed, 
it can be seen in the right plot of Figure 2 that the adaptive wavelet scheme converges at the expected rate or even slightly better. In comparison, we observe the reduced rate $n^{-0.125}$ for uniform refinement. By comparing the norm of the residuals for both strategies, we again confirm the superiority of the adaptive code. In order for uniform refinement to produce an error of $2.9 \cdot 10^{-1}$, it needs more that 3 million degrees of freedom, whereas the adaptive code produces an error of $2.7 \cdot 10^{-1}$ with less than 15000 degrees of freedom. The approximate density, as well as the adaptive refinement, for this example finally are found in Figure 4.

\subsection{Cartoon function as right-hand side}

In the last example, the cube $\Omega:=(-1,1)^{3}$ is chosen as domain, the boundary of which is represented by 6 patches. The right-hand side under consideration is a cartoon function, namely

$$
g(x):= \begin{cases}1 & \text { if }|x-(0,0,1)|^{2} \leq \frac{1}{2} \\ 0, & \text { elsewhere }\end{cases}
$$

Cartoon functions have been studied in [17]. They can be approximated adaptively in an isotropic setting at the rate of $n^{-0.5}$. Since, however, cartoon functions $g$ have a jump discontinuity, the best we can expect is $g \in H^{1 / 2-\varepsilon}(\partial \Omega)$ with $\varepsilon>0$ being arbitrary small. Therefore, Proposition 2.1 implies that the Sobolev regularity of the exact solution $u \in L_{2}(\partial \Omega)$ to the second kind Fredholm integral equation (2) for the double layer potential operator (3) with right-hand side $g$ is also bounded by $1 / 2$. Consequently, for the uniform code, we would expect the approximation rate $n^{-0.25}$. This is in perfect accordance with our numerical experiment; see Figure 5. Moreover, also in this example the observed rate for adaptive refinement is twice as large, i.e., the norm of the residuum decays at a rate of $n^{-0.5}$. Indeed, as seen in the left image of Figure 6, the approximate density is basically also a cartoon function. This issues from the fact that the kernel of the double 
layer operator is zero on a plane patch since $x-y$ is perpendicular to the normal $\eta(y)$. The right image of Figure 6 illustrates the corresponding refinement produced by the adaptive algorithm. It is clearly seen that refinement mainly takes place at the jump of the right-hand side.

\section{Conclusion}

In this article, we considered the adaptive solution of boundary integral equations by using wavelet bases. We have presented the regularity theory which is available and presented numerical results which are in good agreement with the theoretical predictions. In conclusion, we have seen that the adaptive wavelet BEM is able to produce optimal convergence rates, which, for the examples under consideration, are twice as high as for methods based on uniform refinement.

\section{Appendix}

Here we calculate the weighted and unweighted Sobolev regularity of a point singularity. To do so, we need the following auxiliary estimate:

Lemma A.1. Let $d \in \mathbb{N}, \alpha>0$ and $M>2$. Then for all $x, h \in \mathbb{R}^{d}$ with $x_{j}, h_{j} \geq 0$ for every $j=1, \ldots, d$ and $0<|x| \leq|h| / M$ we have

$$
\left.|| x\right|^{-\alpha}-\left.|x+h|^{-\alpha}\left|\geq\left(M^{\alpha}-2^{\alpha}\right)\right| h\right|^{-\alpha}
$$

Proof. Since $x_{j}, h_{j} \geq 0$ for all $j=1, \ldots, d$ we have $|x| \leq|x+h|$ which yields $|x|^{-\alpha} \geq|x+h|^{-\alpha}$ because of $\alpha>0$. This gives

$$
\left.|h|^{\alpha} \cdot|| x\right|^{-\alpha}-|x+h|^{-\alpha} \mid=\left(\frac{|x|}{|h|}\right)^{-\alpha}-\left(\frac{|x+h|}{|h|}\right)^{-\alpha} \geq M^{\alpha}-\left(\frac{|h|}{|x+h|}\right)^{\alpha}
$$


since $|x| \leq|h| / M$ implies $|x|^{\alpha} \leq(|h| / M)^{\alpha}$ and $(|x| /|h|)^{-\alpha} \geq M^{\alpha}$. Now the triangle inequality combined with $|x| /|x+h| \leq 1$ shows that the latter quantity is lower bounded by

$$
M^{\alpha}-\left(\frac{|h|}{|x+h|}\right)^{\alpha} \geq M^{\alpha}-\left(\frac{|x+h|+|x|}{|x+h|}\right)^{\alpha}=M^{\alpha}-\left(1+\frac{|x|}{|x+h|}\right)^{\alpha} \geq M^{\alpha}-2^{\alpha}
$$

and the proof is complete.

Now the regularity result reads as follows.

Proposition A.2. Let $\partial \Omega$ denote a Lipschitz surface according to Subsection 2.1 and assume $\nu$ to be one of its vertices. Moreover, given $1 / 2 \leq \alpha<1$, consider the restriction of

$$
g(x):=|x-\nu|^{-\alpha}, \quad x \in \mathbb{R}^{3},
$$

to $\partial \Omega$. Then

$$
g \in H^{s}(\partial \Omega) \cap X_{\varrho}^{1}(\partial \Omega) \quad \text { for all } \quad 0 \leq s<1-\alpha \quad \text { and all } \quad 0 \leq \varrho<1-\alpha
$$

but $g \notin H^{s}(\partial \Omega)$ if $s \geq 1-\alpha$.

Proof. Step 1. Here we show that (the restriction of) $g$ is contained in $H^{s}(\partial \Omega)$. Note that $\alpha \geq 1 / 2$ implies $0 \leq s<1 / 2$, i.e., it suffices to prove that $g$ belongs to each Sobolev space w.r.t. every single patch $F$ that is part of the description of $\partial \Omega$. According to the structure of $g$ it is obvious that $g \in H^{1}(F) \hookrightarrow H^{s}(F)$ for all patches $F$ which do not contain the critical vertex $\nu$ since $g$ is bounded and smooth on the closure of these patches.

By construction each of the remaining patches $F$ (with $\nu \in \bar{F}$ ) are contained in some face $\Gamma$ of 
the infinite tangent cone $\mathcal{C}$ subordinate to the vertex $\nu$. W.l.o.g. we can assume that $\nu=0$ and

$$
\Gamma=\left\{y=(r \cos (\phi), r \sin (\phi)) \in \mathbb{R}^{2} \mid r>0, \phi \in(0, \gamma)\right\}
$$

with some opening angle $\gamma \in(0,2 \pi)$. Then the restriction of $g$ to $F$ takes the form

$$
g(y)=r^{-\alpha}, \quad y \in F \subset \Gamma .
$$

For $s \geq 0$ and $d \in \mathbb{N}$ it is well-known that $H^{s}(M)=F_{2,2}^{s}(M)=B_{2,2}^{s}(M)$, where $M$ can be any (bounded or unbounded) Lipschitz domain in $\mathbb{R}^{d}$ or $\mathbb{R}^{d}$ itself. Here $B_{p, q}^{s}$ and $F_{p, q}^{s}$ (where $0<p, q<\infty$ and $s \in \mathbb{R}$ ) denote the scales of classical Besov and Triebel-Lizorkin spaces, respectively; see, e.g., [39, Subsection 1.11.1] for precise definitions. Hence, in order to prove the claim, it suffices to show that $g \in F_{2,2}^{s}(F)$. According to the definition of this space it is enough to find some $\widetilde{g} \in F_{2,2}^{s}\left(\mathbb{R}^{2}\right)$ which extends $g$ from $F$ to the whole of $\mathbb{R}^{2}$. To this end, we choose a sufficiently smooth cut-off function $\zeta: \mathbb{R} \rightarrow[0,1]$ with

$$
\zeta(r)= \begin{cases}1 & \text { if } 0 \leq r<R, \\ 0 & \text { if } 2 R \leq r,\end{cases}
$$

and $R>0$ large enough such that $\zeta(|y|) \equiv 1$ for all $y \in F$. Then

$$
\widetilde{g}(y):=|y|^{-\alpha} \zeta(|y|), \quad y \in \mathbb{R}^{2},
$$

defines an extension of $g$, i.e., it satisfies $\left.\widetilde{g}\right|_{F}=g$. Hence, it only remains to prove the membership of $\widetilde{g}$ in $F_{2,2}^{s}\left(\mathbb{R}^{2}\right)$. Fortunately, $\widetilde{g}$ with $\alpha \neq 0$ coincides with the function $f_{-\alpha, 0}$ studied in [36, Subsection 2.3.1] and there it is shown that for $s>2 \cdot \max \{0,1 / p-1\}$ and $0<p, q<\infty$ we indeed have $\widetilde{g}=f_{-\alpha, 0} \in F_{p, q}^{s}\left(\mathbb{R}^{2}\right)$ if and only if $s<2 / p-\alpha$. This particularly shows that $0<\alpha<1$ implies 
$\tilde{g} \in F_{2,2}^{s}\left(\mathbb{R}^{2}\right)$ for all $0<s<1-\alpha$. But then a simple embedding shows that the case $s=0$ is covered as well. So, the proof of $g \in H^{s}(\partial \Omega)$ for all $0 \leq s<1-\alpha$ is complete.

Step 2. We turn to the proof of the membership of $g$ in $X_{\varrho}^{1}(\partial \Omega)$. Here we have to show that $\varphi_{n} g \in X_{\varrho}^{1}\left(\partial \mathcal{C}_{n}\right), n=1, \ldots, N$, where $\partial \mathcal{C}_{n}$ is the boundary of the infinite tangent cone subordinate to the $n$th vertex $\nu_{n}$ and $\varphi_{n}$ is a smooth cut-off function with support in a neighborhood of $\nu_{n}$. For this purpose, we have to bound the norm $\left\|\varphi_{n} g \mid X_{\varrho}^{1}\left(\partial \mathcal{C}_{n}\right)\right\|$ given by (8) with $f_{n}$ replaced by $\varphi_{n} g$ and $k=1$. Due to Step 1 we have $g \in L_{2}(\partial \Omega)$ and hence $\left\|\varphi_{n} g \mid L_{2}\left(\partial \mathcal{C}_{n}\right)\right\|<\infty$ for all $n=1, \ldots, N$. Thus, it remains to estimate

$$
\sum_{\left(\beta_{r}, \beta_{\phi}\right) \in\{(1,0),(0,1)\}}\left\|\left(1+\frac{1}{r}\right)^{\varrho} r^{\beta_{r}} q^{1-\varrho} \frac{\partial}{\partial \phi^{\beta_{\phi}} \partial r^{\beta_{r}}}\left(\varphi_{n} g\right)_{n, t} \mid L_{2}\left(\Gamma^{n, t}\right)\right\|
$$

for all vertices $\nu_{n}, n=1, \ldots, N$, and each face $\Gamma^{n, t}, t=1, \ldots, T_{n}$, of their associated tangent cones. So let $n$ and $t$ be fixed. In order to simplify notation we can again assume that $\Gamma=\Gamma^{n, t}$ is given by $(26)$. If $\nu_{n} \neq \nu$, then

$$
G:=\frac{\partial}{\partial \phi^{\beta_{\phi}} \partial r^{\beta_{r}}} \varphi_{n} g
$$

is smooth on $\bar{\Gamma}$ and thus uniformly bounded. Due to the compact support of $\varphi_{n} g$ and the fact that $q(\phi) \in(0, \pi)$, we obtain

$$
\begin{aligned}
\left\|\left(1+\frac{1}{r}\right)^{\varrho} r^{\beta_{r}} q^{1-\varrho} G \mid L_{2}(\Gamma)\right\|^{2} & \leq\|G\|_{\infty}^{2} \int_{0}^{\gamma} q(\phi)^{2(1-\varrho)} \int_{0}^{R}\left|\left(1+\frac{1}{r}\right)^{\varrho} r^{\beta_{r}}\right|^{2} r \mathrm{~d} r \mathrm{~d} \phi \\
& \lesssim \int_{0}^{R}(1+r)^{2 \varrho} r^{1-2 \varrho+2 \beta_{r}} \mathrm{~d} r<\infty
\end{aligned}
$$

for all $\beta=\left(\beta_{r}, \beta_{\phi}\right) \in \mathbb{N}_{0}^{2}$ provided that $0 \leq \varrho \leq 1 / 2$.

Now, if $\nu_{n}=\nu$, let us define $\Gamma_{0}:=\Gamma \cap\left\{y \in \mathbb{R}^{2}|| y \mid<R_{0}\right\}$ with $R_{0} \in(0, R)$ chosen small enough 
such that $\varphi_{n} \equiv 1$ in a neighborhood of $\Gamma_{0}$. Then

$$
\begin{aligned}
\left\|\left(1+\frac{1}{r}\right)^{\varrho} r^{\beta_{r}} q^{1-\varrho} \frac{\partial}{\partial \phi^{\beta_{\phi}} \partial r^{\beta_{r}}} \varphi_{n} g \mid L_{2}(\Gamma)\right\|^{2}= & \left\|\left(1+\frac{1}{r}\right)^{\varrho} r^{\beta_{r}} q^{1-\varrho} \frac{\partial}{\partial \phi^{\beta_{\phi}} \partial r^{\beta_{r}}} g \mid L_{2}\left(\Gamma_{0}\right)\right\|^{2} \\
& +\left\|\left(1+\frac{1}{r}\right)^{\varrho} r^{\beta_{r}} q^{1-\varrho} \frac{\partial}{\partial \phi^{\beta_{\phi}} \partial r^{\beta_{r}}} \varphi_{n} g \mid L_{2}\left(\Gamma \backslash \Gamma_{0}\right)\right\|^{2} .
\end{aligned}
$$

Note that for $\Gamma \backslash \Gamma_{0}$ the same arguments as above apply. Moreover, if $\beta=\left(\beta_{r}, \beta_{\phi}\right)=(0,1)$, then the first summand vanishes due to the rotational invariance of $g$, i.e., $\partial g / \partial \phi \equiv 0$ as $g$ does not depend on the angular variable $\phi$. So we are left with bounding the first term for $\beta=(1,0)$. Here we have

$$
\begin{aligned}
\int_{0}^{\gamma} & \int_{0}^{R_{0}}\left|\left(1+\frac{1}{r}\right)^{\varrho} r q(\phi)^{1-\varrho} \frac{\partial}{\partial r} r^{-\alpha}\right|^{2} r \mathrm{~d} r \mathrm{~d} \phi \\
\quad & \int_{0}^{\gamma} q(\phi)^{2(1-\varrho)} \mathrm{d} \phi \int_{0}^{R_{0}}\left(1+\frac{1}{r}\right)^{2 \varrho} r^{3}\left|-\alpha r^{-\alpha-1}\right|^{2} \mathrm{~d} r \\
& \lesssim \int_{0}^{R_{0}}(1+r)^{2 \varrho} r^{1-2 \alpha-2 \varrho} \mathrm{d} r<\infty
\end{aligned}
$$

provided that $0 \leq \varrho \leq 1$ and $1-2 \alpha-2 \varrho>-1$ which particularly holds under the given assumptions $0 \leq \varrho<1-\alpha$ and $1 / 2 \leq \alpha<1$.

Step 3. Finally, we show that $g \notin H^{s}(\partial \Omega)$ if $s \geq 1-\alpha$. Since $0<1-\alpha \leq 1 / 2$ it suffices to prove $g \notin H^{1-\alpha}(F)=B_{2,2}^{1-\alpha}(F)$ for some patch $F \subset \partial \Omega$. To do so, let $F$ denote a patch with $\nu \in \bar{F}$ and let $\Gamma$ be the face of the corresponding tangent cone associated to $\nu$ which contains $F$. W.l.o.g. we again assume that $\nu=0$ and that $\Gamma$ is given by (26). Then we can choose $T>0$ small enough and $M>2$ large enough such that for all $h \in H_{T, M}:=\left\{\in \mathbb{R}^{2}\left|h_{1}, h_{2} \geq 0,\right| h \mid \leq T\right\}$ we have

$$
\Gamma_{h}:=\Gamma \cap\left\{y \in \mathbb{R}^{2}\left|x_{1}, x_{2} \geq 0,\right| y \mid<\frac{|h|}{M}\right\} \subseteq F_{h}:=\{y \in F \mid y+h \in F\} \subseteq F .
$$

Then Lemma A.1 yields that for all $h \in H_{T, M}$ the first order finite difference $\Delta_{h} g:=g(\cdot+h)-g$ 
satisfies

$$
\begin{aligned}
\left\|\Delta_{h} g \mid L_{2}\left(\Gamma_{h}\right)\right\|^{2} & =\int_{\Gamma_{h}}\left|\Delta_{h} g(y)\right|^{2} \mathrm{~d} y \\
& =\left.\int_{\Gamma_{h}}|| y\right|^{-\alpha}-\left.|y+h|^{-\alpha}\right|^{2} \mathrm{~d} y \\
& \geq\left(M^{\alpha}-2^{\alpha}\right)^{2}|h|^{-2 \alpha} \int_{0}^{\min \{\gamma, \pi / 2\}} \int_{0}^{|h| / M} r \mathrm{~d} r \mathrm{~d} \phi \\
& \sim_{M, \alpha, \gamma}|h|^{2-2 \alpha}
\end{aligned}
$$

and hence for the modulus of smoothness it holds

$$
\omega(g, t, F)_{2}:=\sup _{\substack{h \in \mathbb{R}^{2} \\|h| \leq t}}\left\|\Delta_{h} g\left|L_{2}\left(F_{h}\right)\left\|\geq \sup _{\substack{h \in \mathbb{R}^{2} \\ h_{1}, h_{2} \geq 0,|h| \leq t}}\right\| \Delta_{h} g\right| L_{2}\left(\Gamma_{h}\right)\right\| \gtrsim t^{1-\alpha} \quad \text { for all } \quad 0<t<T
$$

where we used that $1-\alpha>0$. This shows

$$
\left\|g\left|H^{1-\alpha}(F)\|\sim\| g\right| B_{2,2}^{1-\alpha}(F)\right\|:=\int_{0}^{\infty}\left[t^{-(1-\alpha)} \omega(g, t, F)_{2}\right]^{2} \frac{\mathrm{d} t}{t} \gtrsim \int_{0}^{T} \frac{\mathrm{d} t}{t}=\infty
$$

and thus $g \notin H^{s}(\partial \Omega)$ if $s \geq 1-\alpha$.

\section{References}

[1] M. Bebendorf. Approximation of boundary element matrices. Numer. Math., 86:565-589, 2000.

[2] C. Canuto, A. Tabacco, and K. Urban. The wavelet element method part I: Construction and analysis. Appl. Comput. Harmon. Anal., 6(1):1-52, 1999.

[3] C. Canuto, A. Tabacco, and K. Urban. The wavelet element method part II: Realization and additional features in 2D and 3D. Appl. Comput. Harmon. Anal., 8(2):123-165, 2000. 
[4] A. Cohen, W. Dahmen, and R. A. DeVore. Adaptive wavelet methods for elliptic operator equations: Convergence rates. Math. Comp., 70:27-75, 2001.

[5] A. Cohen, W. Dahmen, and R. A. DeVore. Adaptive wavelet methods II: Beyond the elliptic case. Found. Comput. Math., 2(3):203-245, 2002.

[6] A. Cohen and R. Masson. Wavelet adaptive method for second order elliptic problems: Boundary conditions and domain decomposition. Numer. Math., 86(2):193-238, 2000.

[7] B. E. Dahlberg and C. E. Kenig. Hardy spaces and the Neumann problem in $L^{p}$ for Laplace's equation in Lipschitz domains. Ann. Math. (2), 125:437-465, 1987.

[8] S. Dahlke, W. Dahmen, and R. A. Devore. Nonlinear approximation and adaptive techniques for solving elliptic operator equations. In W. Dahmen, A. Kurdila, and P. Oswald, editors, Multsicale Wavelet Methods for Partial Differential Equations, pages 237-283, San Diego, 1997. Academic Press.

[9] S. Dahlke and R. A. DeVore. Besov regularity for elliptic boundary value problems. Comm. Partial Differential Equations, 22(1-2):1-16, 1997.

[10] S. Dahlke, E. Novak, and W. Sickel. Optimal approximation of elliptic problems by linear and nonlinear mappings II. J. Complexity, 22(4):549-603, 2006.

[11] S. Dahlke and M. Weimar. Besov regularity for operator equations on patchwise smooth manifolds. Found. Comput. Math., 15(6):1533-1569, 2015.

[12] W. Dahmen, H. Harbrecht, and R. Schneider. Adaptive methods for boundary integral equations: Complexity and convergence estimates. Math. Comp., 76:1243-1274, 1998. 
[13] W. Dahmen, H. Harbrecht, and R. Schneider. Compression techniques for boundary integral equations. Asymptotically optimal complexity estimates. SIAM J. Numer. Anal., 43(6):22512271, 2006.

[14] W. Dahmen and R. Schneider. Composite wavelet bases for operator equations. Math. Comp., 68:1533-1567, 1999.

[15] R. A. DeVore. Nonlinear approximation. Acta Numer., 7:51-150, 1998.

[16] R. A. DeVore, B. Jawerth, and V. Popov. Compression of wavelet decompositions. Am. J. Math., 114(4):737-785, 1992.

[17] D. Donoho. Sparse components of images and optimal atomic decomposition. Constr. Approx., $17: 353-382,2001$.

[18] J. Elschner. The double layer potential operator over polyhedral domains I: Solvability in weighted Sobolev spaces. App. Anal., 45:117-134, 1992.

[19] B. Faermann. Localization of the Aronszajn-Slobodeckij norm and application to adaptive boundary element methods. Part II: The three-dimensional case. Numer. Math., 92(3):467-499, 2002.

[20] M. Feischl, M. Karkulik, J. Melenk, and D. Praetorius. Quasi-optimal convergence rate for an adaptive boundary element method. SIAM J. Numer. Anal., 51(2):1327-1348, 2013.

[21] M. Frazier and B. Jawerth. A discrete transform and decomposition of distribution spaces. J. Funct. Anal., 93(1):297-318, 1990.

[22] T. Gantumur. An optimal adaptive wavelet method for nonsymmetric and indefinite elliptic problems. J. Comput. Appl. Math., 211(1):90-102, 2008. 
[23] T. Gantumur. Adaptive boundary element methods with convergence rates. Numer. Math., 124:471-516, 2013.

[24] T. Gantumur, H. Harbrecht, and R. Stevenson. An optimal adaptive wavelet method for elliptic equations without coarsening. Math. Comput., 76:615-629, 2007.

[25] T. Gantumur and R. Stevenson. Computation of singular integral operators in wavelet coordinates. Computing, 76:77-107, 2006.

[26] W. Hackbusch. Elliptic Differential Equations: Theory and Numerical Treatment, volume 18 of Springer Series in Computational Mathematics. Springer, Berlin, 1992.

[27] W. Hackbusch and Z. Nowak. On the fast matrix multiplication in the boundary element method by panel clustering. Numer. Math., 54:463-491, 1989.

[28] H. Harbrecht and R. Schneider. Biorthogonal wavelet bases for the boundary element method. Math. Nachr., 269-270:167-188, 2004.

[29] H. Harbrecht and R. Stevenson. Wavelets with patchwise cancellation properties. Math. Comp., 75:1871-1889, 2006.

[30] H. Harbrecht and M. Utzinger. On adaptive wavelet boundary element methods. Technical Report 2015-42, Mathematisches Institut, Universität Basel, Switzerland, 2015. To appear in $J$. Comput. Math.

[31] D. S. Jerison and C. E. Kenig. The Dirichlet problem in non-smooth domains. Ann. Math. (2), 113:367-382, 1981.

$[32]$ D. S. Jerison and C. E. Kenig. The inhomogeneous Dirichlet problem in Lipschitz domains. J. Funct. Anal., 130(1):161-219, 1995. 
[33] N. Kalton, S. Mayboroda, and M. Mitrea. Interpolation of Hardy-Sobolev-Besov-TriebelLizorkin spaces and applications to problems in partial differential equations. In L. De Carli and M. Milman, editors, Interpolation Theory and Applications (Contemporary Mathematics 445), pages 121-177, Providence, RI, 2007. Amer. Math. Soc.

[34] C. E. Kenig. Harmonic Analysis Techniques for Second Order Elliptic Boundary Value Problems, volume 83 of Regional Conference Series in Mathematics. Amer. Math. Soc., Providence, RI, 1994.

[35] V. Rokhlin. Rapid solution of integral equations of classical potential theory. J. Comput. Phys., 60:187-207, 1985.

[36] T. Runst and W. Sickel. Sobolev Spaces of Fractional Order, Nemytskij Operators and Nonlinear Partial Differential Equations. de Gruyter, Berlin, 1996.

[37] S. A. Sauter and C. Schwab. Boundary Element Methods, volume 39 of Springer Series in Computational Mathematics. Springer, Berlin, 2011.

[38] R. Schneider. Multiskalen-und Wavelet-Matrixkompression: Analysisbasierte Methoden zur effizienten Lösung großer vollbesetzter Gleichungssysteme. Teubner, 1998.

[39] H. Triebel. Theory of Function Spaces III. Birkhäuser, Basel, 2006.

[40] M. Utzinger. An Adaptive Wavelet Method for the Solution of Boundary Integral Equations in Three Dimensions. PhD thesis, Universität Basel, 2016.

[41] G. Verchota. Layer potentials and regularity for the Dirichlet problem for Laplace's equation in Lipschitz domains. J. Funct. Anal., 59:572-611, 1984.

[42] M. Weimar. Almost diagonal matrices and Besov-type spaces based on wavelet expansions. J. Fourier Anal. Appl., 22(2):251-284, 2016. 


\title{
Stephan Dahlke
}

Philipps-University Marburg

Faculty of Mathematics and Computer Science

Workgroup Numerics and Optimization

Hans-Meerwein-Straße, Lahnberge

35032 Marburg, Germany

dahlke@mathematik.uni-marburg.de

\section{Helmut Harbrecht and Manuela Utzinger}

University of Basel

Department of Mathematics and Computer Science

Research Group of Computational Mathematics

Spiegelgasse 1

4051 Basel, Switzerland

\{helmut.harbrecht, manuela.utzinger\}@unibas.ch

\author{
Markus Weimar \\ Ruhr-Universität Bochum \\ Faculty of Mathematics \\ Workgroup Numerics \\ Universitätsstrae 150 \\ 44801 Bochum, Germany \\ markus.weimar@rub.de
}



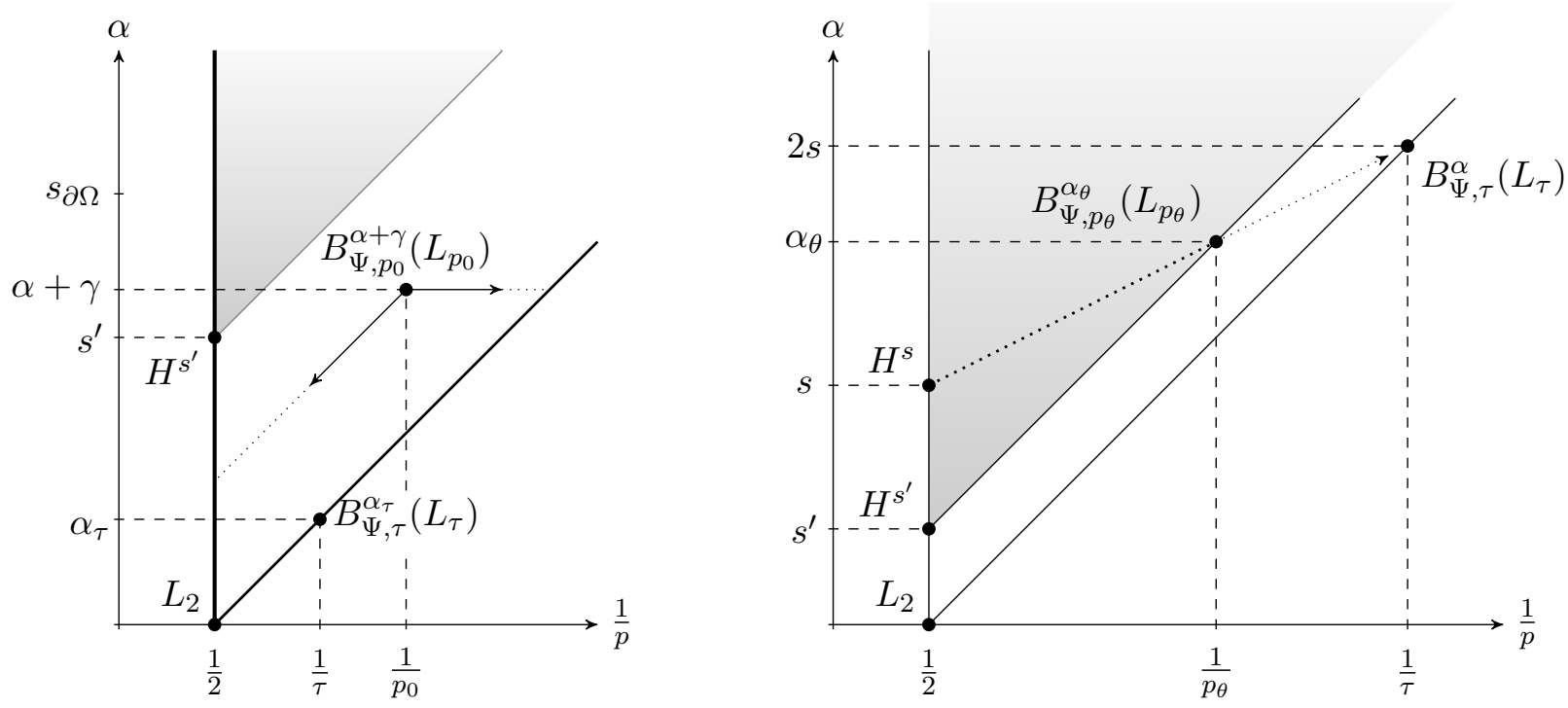

Figure 1: DeVore-Triebel diagrams visualizing the area of admissible parameters, as well as embeddings related to Proposition 3.2 (left), and Theorem 3.4 (right).
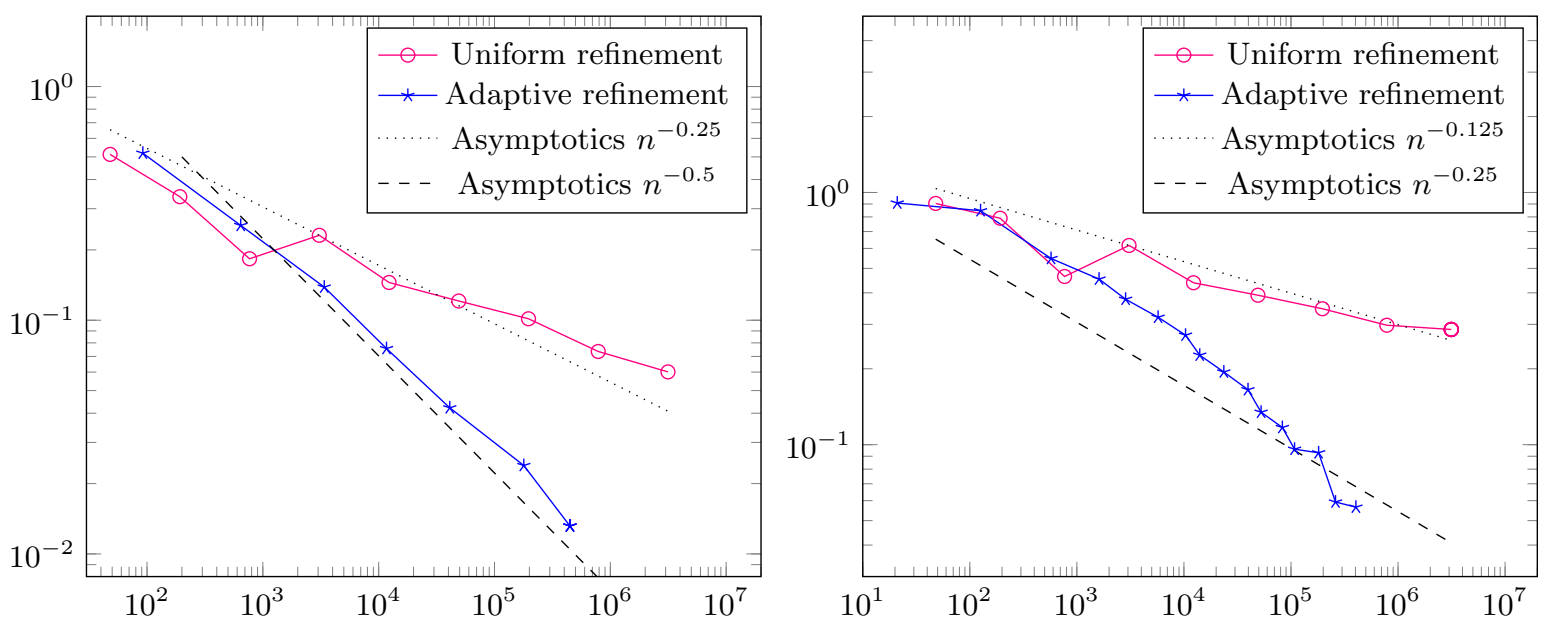

Figure 2: Energy norm of the residual vector for adaptive and uniform refinement for $\alpha=0.5$ (left) and $\alpha=0.75$ (right). 

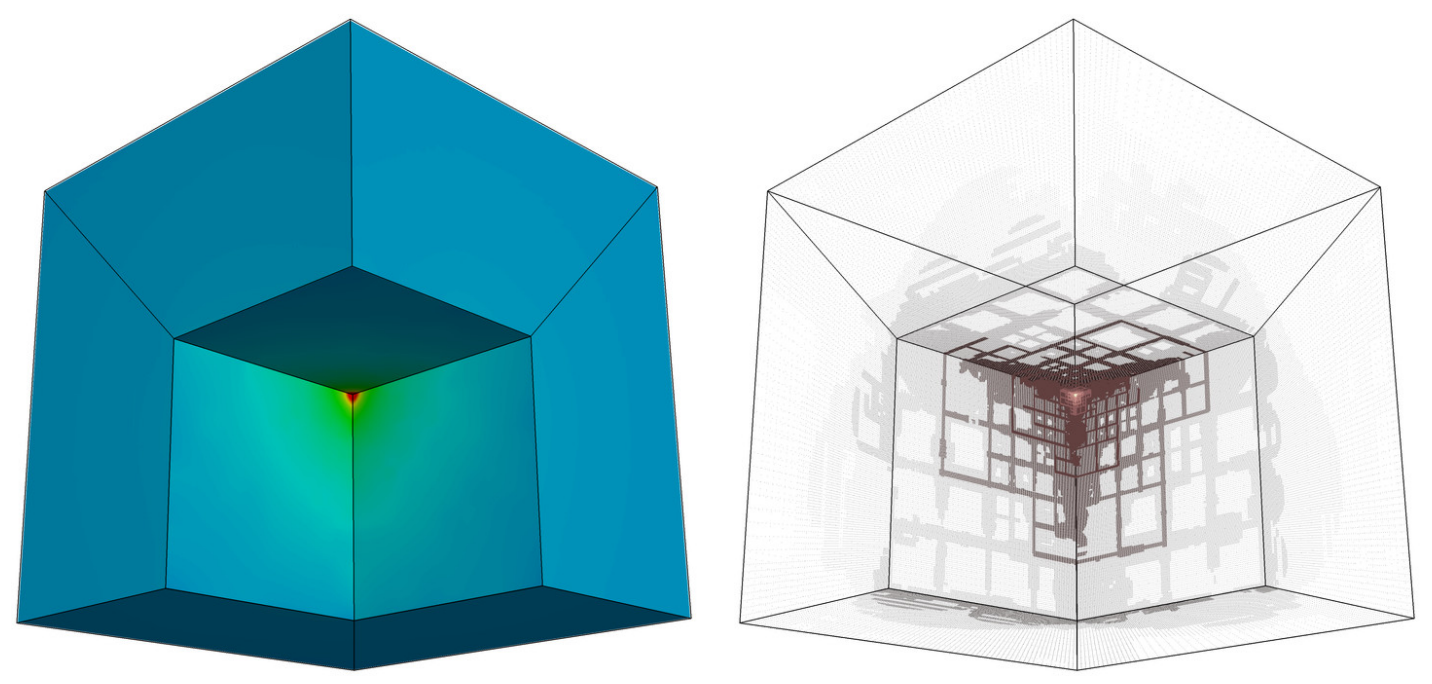

Figure 3: Approximate density $u$ (left) and associated adaptive refinement (right) for $\alpha=0.5$.
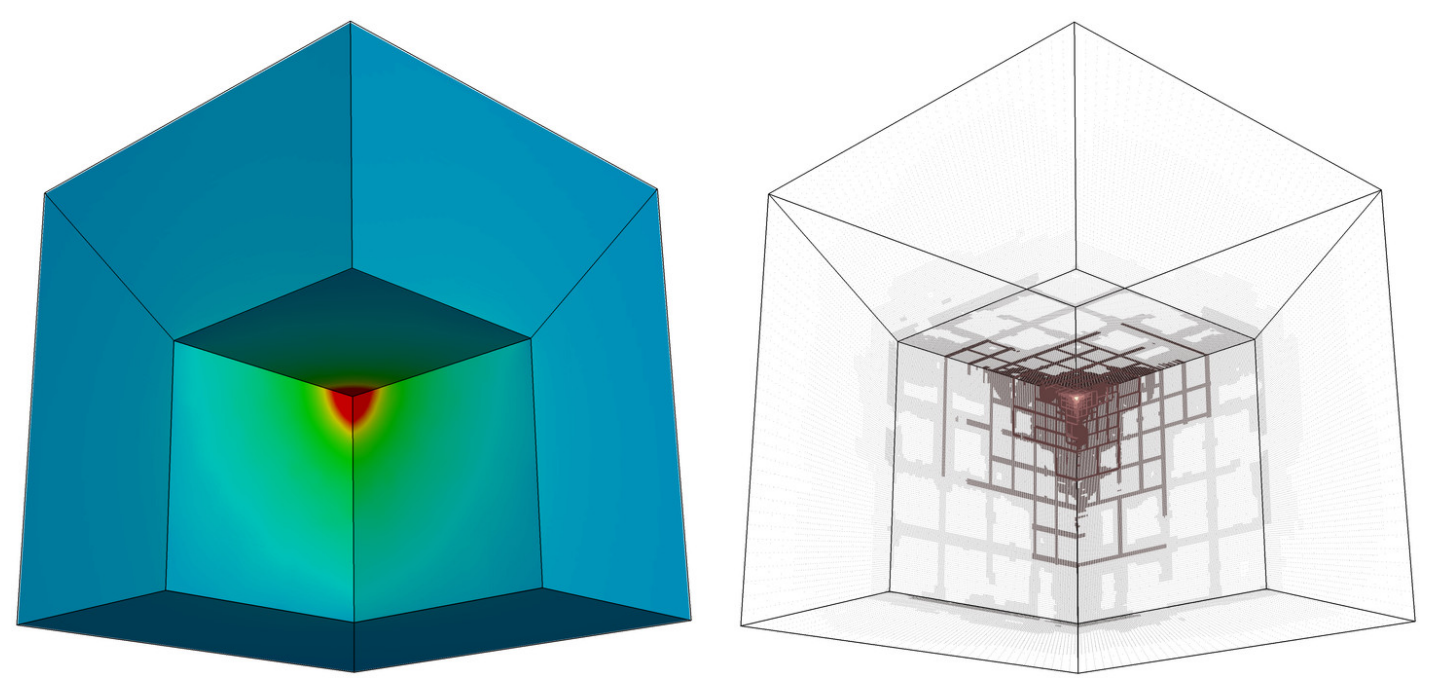

Figure 4: Approximate density $u$ (left) and associated adaptive refinement (right) for $\alpha=0.75$. 


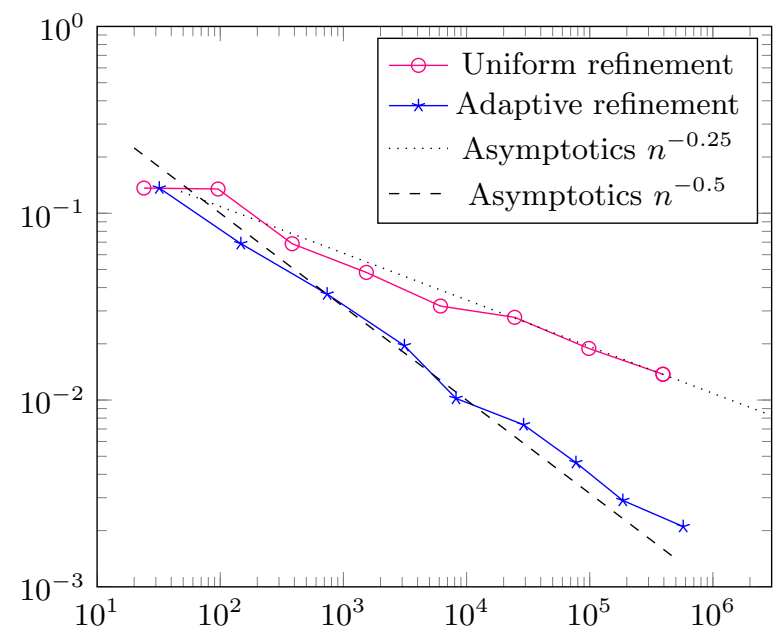

Figure 5: Energy norm of the residual for adaptive and uniform refinement for the cartoon right-hand side.
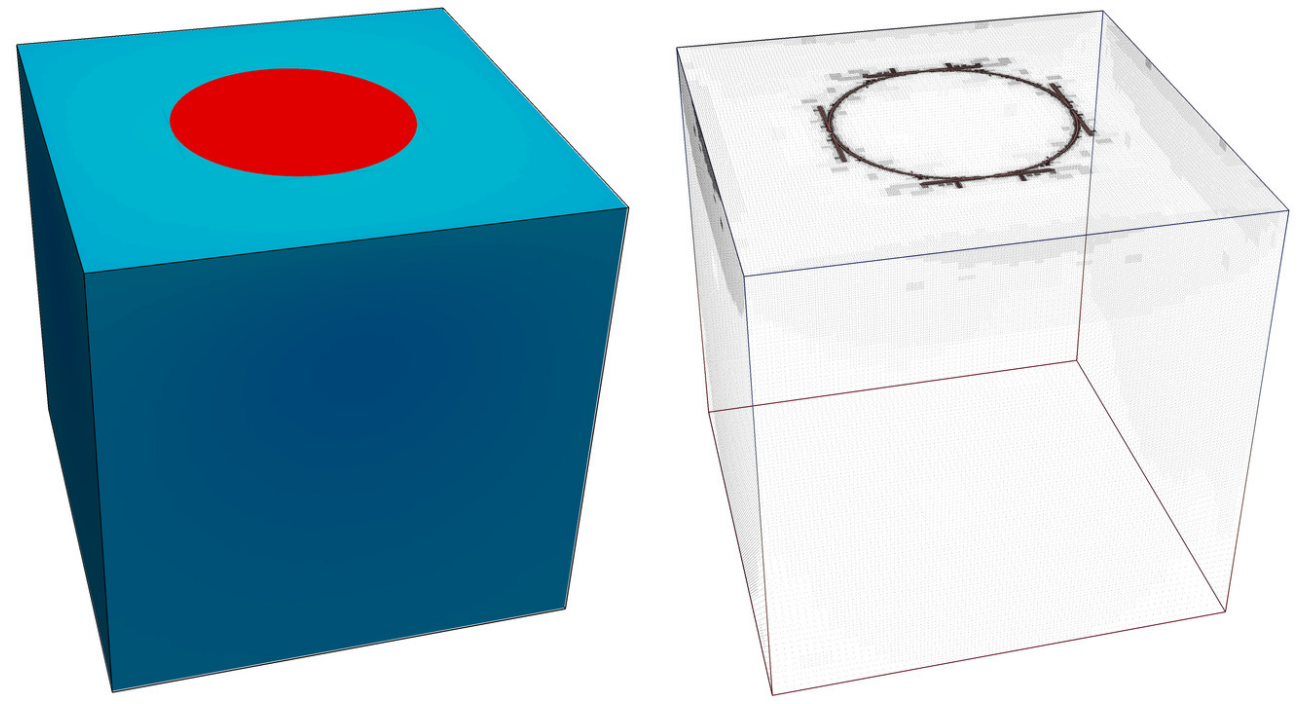

Figure 6: Approximate density $u$ (left) and associated refinement (right) for the cartoon right-hand side on the cube. 